\title{
TIP60/P400/H4K12ac Plays a Role as a Heterochromatin Back-up Skeleton in Breast Cancer
}

\author{
MOUHAMED IDRISSOU ${ }^{1,2}$, TIPHANIE BOISNIER ${ }^{1,2}$, ANNA SANCHEZ ${ }^{1,2}$, FATMA ZOHRA HOUFAF KHOUFAF ${ }^{1,2}$, \\ FREDERIQUE PENAULT-LLORCA ${ }^{2,3}$, YVES-JEAN BIGNON ${ }^{1,2}$ and DOMINIQUE BERNARD-GALLON ${ }^{1,2}$ \\ ${ }^{I}$ Department of Oncogenetics, Centre Jean Perrin, CBRV, Clermont-Ferrand, France; \\ ${ }^{2}$ INSERM-UMR 1240-Imagerie Moléculaire et Stratégies Théranostiques (IMoST), Clermont-Ferrand, France; \\ ${ }^{3}$ Department of Biopathology, Centre Jean Perrin, Clermont-Ferrand, France
}

\begin{abstract}
Background/Aim: In breast cancer, initiation of carcinogenesis leads to epigenetic dysregulation, which can lead for example to the loss of the heterochromatin skeleton SUV39H1/H3K9me3/HP1 or the supposed secondary skeleton TIP60/P400/H4K12ac/BRD (2/4), which allows the maintenance of chromatin integrity and plasticity. This study investigated the relationship between TIP60, P400 and H4K12ac and their implications in breast tumors. Materials and Methods: Seventy-seven patients diagnosed with breast cancer were included in this study. Chromatin immunoprecipitation (ChIP) assay was used to identify chromatin modifications. Western blot and reverse transcription and quantitative real-time PCR were used to determine protein and gene expression, respectively. Results: We verified the variation in H4K12ac enrichment and the colocalization of H4K12ac and TIP60 on the euchromatin and heterochromatin genes, respectively, by ChIP-qPCR and ChIP-reChIP, which showed an enrichment of H4K12ac on specific genes in tumors compared to the adjacent healthy tissue and a co-localization of H4K12ac with TIP6O in different breast tumor types. Furthermore, RNA and protein expression of TIP60 and P400 was investigated and overexpression of TIP60 and P400 mRNA was associated with tumor aggressiveness. Conclusion: There is a potential interaction between H4K12ac and TIP60 in heterochromatin or euchromatin in breast tumors.
\end{abstract}

This article is freely accessible online.

Correspondence to: Dominique Bernard-Gallon, Department of Oncogenetics, Centre Jean Perrin, CBRV, IMoST, 28 place HenriDunant, 63001 Clermont-Ferrand, France. Tel: +33 0473178358, e-mail: dominique.gallon-bernard@clermont.unicancer.fr

Key Words: Breast cancer tumor, TIP60, H4K12ac, P400, heterochromatin, euchromatin.
Breast cancer is the most frequent cancer $(24.2 \%$ new cases in 2018) and the leading cause of cancer-related death (15\% in 2018) (1). The majority of breast cancers are sporadic (non-hereditary), representing 80 to $90 \%$ of breast cancers (2). Deregulation of chromatin integrity and plasticity is among the causes of sporadic cancer (3). They occur at the epigenetic level in the different compartments of chromatin, which are heterochromatin (compacted chromatin) that inhibits gene expression, and euchromatin (relaxed chromatin) that promotes gene transcription. These chromatin states are maintained by so-called epigenetic proteins, which, by placing groups or marks on the histones, allow entry or exit from one state to another (4-6).

Among these proteins, SUV39H1, which is a histone methyltransferase (HMT) that adds the H3K9me3 marker on the site where the HP1 protein will bind, thus forming the SUV39H1/H3K9me3/HP1 skeleton which keeps the chromatin closed and inhibits transcription in the heterochromatin region $(7,8)$. Heterochromatin houses inactive genes such as numerous oncogenes; the loss of this structure would lead to the transcription of oncogenes. Several studies have shown a loss of the SUV39H1 protein in cancer, but also a loss of the SUV39H1/H3K9me3/HP1 skeleton during the onset of carcinogenesis $(7,9)$.

The acetyl-transferases (HAT) such as TIP60 are able to add acetyl groups on the $\mathrm{N}$-terminal tails of histones resulting in the opening of the chromatin leading to euchromatin (10). TIP60 is also capable of acetylating non-histone proteins such as p53 and ATM allowing their activation (11-13). Many studies have shown that histone acetylation by TIP60 is observed in euchromatin and heterochromatin, allowing the maintenance of these structures (14). Indeed, TIP60 can be recruited at the heterochromatin due to its chromo-domain capable of binding to $\mathrm{H} 3 \mathrm{~K} 9 \mathrm{me} 3$ or $\mathrm{H} 3 \mathrm{~K} 9$ me groups (15), but can also be recruited via its complex with the $\mathrm{H} 2 \mathrm{Az}$ variant into the heterochromatin by the ATPase P400 $(16,17)$. This recruitment would compensate for the loss of the heterochromatin maintenance 
skeleton (SUV39H1/H3K9me3/HP1) by the overexpression of histone demethylase. Grézy et al. found that treatment of SUV39H1/2 and NIH3T3 cells with azacytidine resulted in an enrichment of H4K12ac, TIP60, P400 and BRD2 on the heterochromatin (16). This enrichment revealed the existence of a back-up skeleton for the heterochromatin that comprised TIP60/ H4K12ac/BRD2. Indeed, bromo-domain proteins such as BRD2 and BRD4 are able to bind to the H4K12ac mark preventing the detachment of chromatin (18). In breast cancer, a decrease in the protein levels and a residual activity of TIP60has been observed (19).

In this study, we hypothesized that the H4K12ac modification in heterochromatin and euchromatin is realized by TIP60 and its complex especially with P400, thus allowing maintenance of chromatin stability and integrity. This recruitment of TIP60 could explain its presence in heterochromatin and thus, the dysregulation of the acetylation of p53 and tumor suppressors.

The study of this residual activity of TIP60 in the maintenance of chromatin structures will allow a better understanding of its role in the aggressiveness of cancers and especially of breast cancer.

\section{Materials and Methods}

Clinical samples. This study included 77 patients admitted to the Centre Jean Perrin from September 2008 to February 2019, and diagnosed with breast cancer (Table I). Patients were informed about the study and gave informed consent prior to inclusion. All 77 tumors and their adjacent normal breast tissues were obtained from the Centre Jean Perrin tumor bank, Biological Resource Center (CRB), accredited under No.BB-0033-00075, where they were stored in liquid nitrogen at $-196^{\circ} \mathrm{C}$. The 77 patients selected did not receive chemotherapy and/or radiotherapy, were not predisposed to breast cancer and had no family members with breast cancer.

Chromatin immunoprecipitation (ChIP) assays and Quantitative real-time PCR method and data analysis. ChIP assays were performed on chromatin extracted from breast tumors using the Auto iDeal ChIP-seq kit for Histones (C01010171, Diagenode, Seraing, Belgium) according to manufacturer's instructions and the experimental setup developed in our laboratory $(20,21)$. The antibodies used were $3 \mu \mathrm{g}$ of anti-H4k12ac Abs (C15200218, Diagenode) and $1 \mu \mathrm{g}$ non-immune rabbit $\mathrm{IgG}$ (Kch-504-250, Diagenode) serving as a negative control.

For Re-ChIP assays, the immunoprecipitated DNA from the first ChIP assay was used. The second ChIP assay (re-ChIP) was then carried out using $3 \mu \mathrm{g}$ of anti-TIP60 Abs (SC-166323, Santa Cruz Biotechnology, Dallas, TX, USA) according to the experimental setup developed in our laboratory $(20,21)$. The quality control and efficacy of all Chip assays performed in this study were verified using positive and negative controls provided in the manufacturer's kit and according to their instructions (Diagenode). Control of ChIP analysis was performed prior to direct H4K12ac ChIP assays and prior to H4K12ac and TIP60 ChIP and re-ChIP assays.

Real-time qPCR was performed in triplicate at $25 \mu$ l final reaction volume that included $5 \mu \mathrm{l}$ of IP or input, $1 \times$ de $\mathrm{SYBR}^{\circledR}$
Table I. Characteristics of the breast cancer patients included in this study.

\begin{tabular}{lcrrrrrr}
\hline & \multicolumn{2}{c}{ Age } & \multicolumn{3}{c}{ Grade } & \multicolumn{2}{c}{ Ki67 } \\
\cline { 2 - 8 } & $<50$ & $>50$ & I & II & III & $\leq 20$ & $>20$ \\
\hline Luminal A & 2 & 26 & 7 & 13 & 8 & 18 & 10 \\
Luminal B & 1 & 27 & 19 & 5 & 4 & 17 & 9 \\
TNBC & 1 & 20 & 0 & 6 & 15 & 4 & 17 \\
\hline
\end{tabular}

Green PCR Master Mix (Applied Biosystems, CA, USA), 200 nM for each GAPDH (C17011047, Diagenode) and Myoglobin Exon 2 primers (C17011006, Diagenode), on a 7900 HT Fast Real Time PCR System (Applied Biosystems).

Protein extraction and immunoblot analysis. Breast cancer tumors and their adjacent healthy tissues were crushed with the crusher (french press) and protein extracts were obtained using NucleoSpin ${ }^{\circledR}$ RNA/Protein, Mini kit for RNA and protein purification (MachereyNagel, Hoerdt, France). About 25-40 $\mu$ g of extracted proteins were resolved by electrophoresis on $8-15 \%$ sodium dodecyl sulfate polyacrylamide gel (SDS-PAGE) (Bio-Rad, Hercules, CA, USA). Electro-transfer, blockage of membranes, immunoblotting and immunolabeling were performed using the manufacturer's protocol and the experimental methods developed in our laboratory $(20,22)$. The primary antibodies (Abs) used were: anti-TIP60 Abs (1/500, GTX112197) and anti-P400 Ab (1/500, GTX116689) were purchased from GenTex (CA, USA), anti-H4k12ac Abs (1/750, C15200218, Diagenode) and anti- GAPDH Abs (1/5000, sc-47724, Santa Cruz Biotechnology). The secondary Abs: anti-mouse IgG $(1 / 2000$, S3721) and anti-rabbit IgG (1/2000, S3738) were purchased from Promega (Madison, WI, USA).

RNA extraction, reverse transcription $(R T)$ and quantitative realtime PCR analysis. Breast tumors and their adjacent healthy tissues were crushed at the french press. RNA extracts were obtained using NucleoSpin ${ }^{\circledR}$ RNA/Protein, Mini kit for RNA, protein purification (Macherey-Nagel, France) and reverse transcription were performed using a High Capacity cDNA Reverse Transcription kit (Applied Biosystems) and Synthesized cDNAs were amplified using TaqMan Gene expression PCR Master Mix (Applied Biosystems) according to the manufacturer's protocol and the experimental methods developed in our laboratory $(20,22)$. The TaqMan Gene Expression assay-on-demand used were TIP60 [Hs00197310_m1] or P400 [Hs01566078_m1] and endogenous control 18S Rrna [Hs99999901_s1].

Statistical analyses. Relative expression levels of TIP60, p400 and $\mathrm{H} 4 \mathrm{~K} 12 \mathrm{ac}$ protein assayed by immunoblotting were quantified using ImageJ software. Data analyses were performed using Prism 6 software (GraphPad Software, Inc., La Jolla, CA, USA). Statistical significance was calculated by One-Way ANOVA test with a multiple comparison test to assess statistical significance between groups. A $p$-value $<0.05\left(^{*}\right)$ was considered significant, $p<0.01(* *)$ very significant and a $p<0.001(* * *)$ highly significant, as stated in the figure legends. 

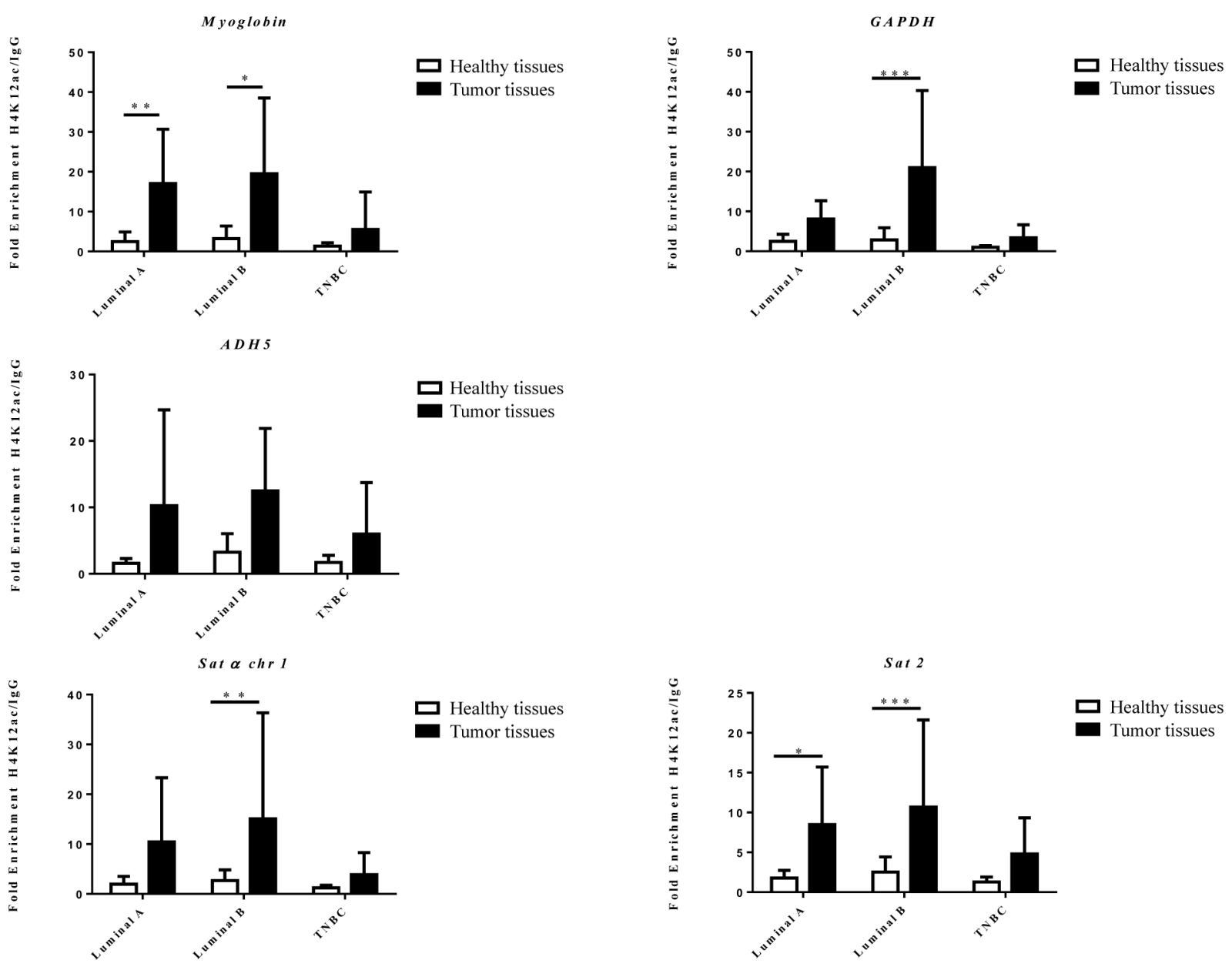

Figure 1. Fold enrichment in H4K12ac over IgG (illustrating the presence of H4K12ac on promoters in euchromatin genes (GAPDH and ADH5) and in heterochromatin (Myoglobin, SAT $\alpha$ and SAT 2 genes) in adjacent healthy breast tissues and Luminal A, Luminal B and TNBC breast tumors. Results are expressed as fold increase $\pm S D$ of $(N=19, N=19$ and $N=12$, for Luminal $A$, Luminal $B$ and TNBC, respectively). p-values were calculated by two-tailed students $t$-test $\left(*\right.$ indicating $p<0.05, *^{*} p<0.01$ and $\left.*^{* *} p<0.001\right)$. Black bar: breast tumors and white bar: healthy breast tissues.

\section{Results}

H4K12ac enriched on genes in euchromatin and heterochromatin in breast tumors. To observe H4K12ac enrichment on the promoters in euchromatin (GAPDH and $A D H 5)$ and heterochromatin (Myoglobin, SAT $\alpha$ and SAT2) genes in breast cancer, a ChIP-qPCR was performed on 19 Luminal A, 19 Luminal B and 12 triple negative breast cancer (TNBC) tumors and their adjacent healthy tissues (Figure 1). An enrichment of H4K12ac was found in different euchromatin and heterochromatin genes in almost equal proportions in healthy breast tissues (Figure 1), whereas this enrichment was significantly increased in Luminal A and B tumors compared to their adjacent healthy tissues (Figure 1). In TNBC tumors, this enrichment increased slightly compared to healthy tissues, and a decrease in enrichment can be seen in TNBC compared to Luminal tumors (Figure 1). These results suggest that there is an equal distribution of H4K12ac in the different chromatin compartments in healthy breast tissues, but this marker is highly increased in Luminal tumors and slightly in TNBC tumors.

Co-localization of H4K12ac and TIP60 in breast tumors. To determine whether TIP60 is responsible for the H4K12ac modification, we performed ChIP-reChIP and examined whether they co-localized in euchromatin (GAPDH and ADH5 genes) and in heterochromatin (Myoglobin, SAT $\alpha$ and SAT 2 genes) (Figure 2). A significant high colocalization was obtained in healthy tissues compared to tumors and this co-localization decreased significantly in luminal A and TNBC tumors. In luminal B tumors, the colocalization of H4K12ac and TIP60 was more important than 

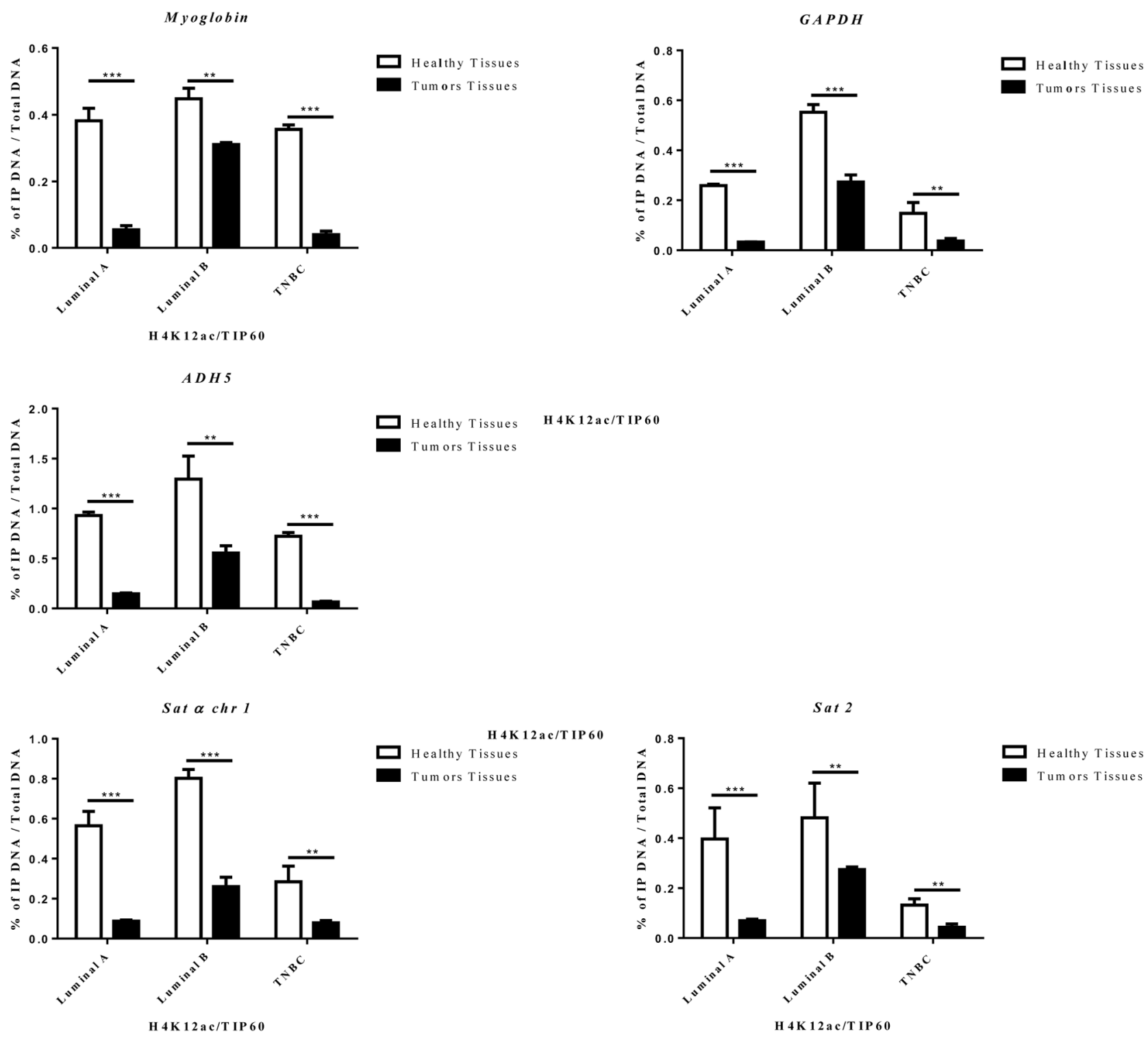

Figure 2. Co-localization of H4K12ac and TIP60 in promoter regions of target genes on euchromatin (GAPDH and ADH5) and heterochromatin (Myoglobin, SATa and SAT 2) in adjacent healthy breast tissues and breast tumors Luminal A, Luminal B and TNBC. Results are expressed as percentage $\pm S D$ of six tumor and healthy tissues. p-values calculated by two-tailed students t-test ( $*$ indicating $p<0.05, * * p<0.01$ and $* * * p<0.001)$. Black bar: breast tumors and white bar: healthy breast tissues.

in other types of tumors. This could suggest that TIP60 is recruited during the carcinogenesis induction, because in the less aggressive tumors there is an increase in H4K12ac mark and co-localization of TIP60 and H4K12ac from Luminal A to Luminal B tumors. This recruitment is lost in the most aggressive TNBC tumors, which could be due to the high decrease in TIP60 expression in these aggressive tumors.

Variations in the expression of TIP60 and P400 in breast tumors. After observing the variations in the enrichment of the H4K12ac mark and the co-localization of TIP60 with this mark, TIP60 and P400 mRNA and protein accumulation in breast tumors and adjacent healthy tissues was examined. RNA expression was studied by RT-qPCR, which demonstrated a significantly lower expression of the mRNA of TIP60 and P400 in Luminal tumors, while a significant overexpression of TIP60 and P400 mRNA was observed in TNBCs tumors (Figure 3). The expression of TIP60 and P400 proteins as well as the H4K12ac modification in breast tumors and their adjacent healthy tissues were studied by 


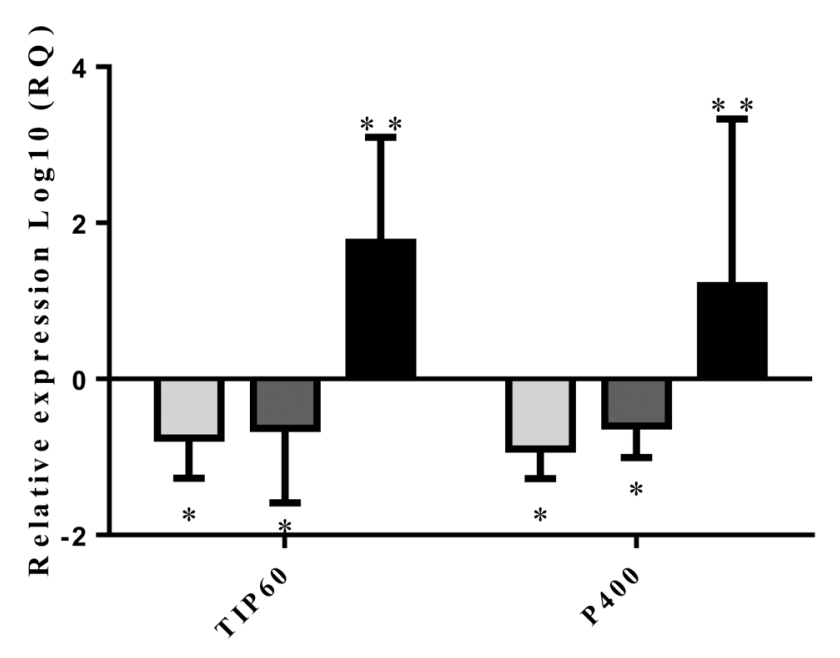

m R N A

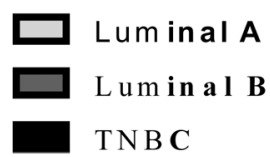

Figure 3. Relative RNA expression of TIP60 and P400 of breast tumors (Luminal A, Luminal B and TNBC) compared to their healthy adjacent tissues. Results are expressed as relative expression Log10 $\pm S D$ of triplicates, the significance is represented by $(*$ if the expression is twice more or less important or $* *$ if the expression is ten times more or less important). Black bar: TNBC breast tumors, grey bar: Luminal B breast tumors and white bar: Luminal A breast tumors.

western blot (Figure 4). A decrease in protein and mark levels between tumors and healthy tissues were observed. These observations demonstrated a difference in the transcription and translation of TIP60 and P400. In fact, transcription of TIP60 and P400 was decreased in the least aggressive tumors (Luminal) and increased in the most aggressive tumors (TNBC) (Figure 3). However, their translation into proteins progressively decreased with the aggressiveness of the tumor (Figure 4). This may suggest that there is a deregulation of the translation of these proteins confirming the decrease in the co-localization between H4K12ac and TIP60 observed by re-ChIP.

\section{Discussion}

In breast cancer, downregulation of TIP60 is observed early in carcinogenesis $(19,23)$, which seems to affect the activities of TIP60 in cancer. TIP60 is a pleiotropic protein that plays a role in several cellular mechanisms involved in tumor development and growth due to its ability to acetylate non-histone proteins such as p53 and $\operatorname{ATM}(12,13,24)$.
Recruitment of TIP60 in heterochromatin to compensate for the loss of the SUV39H1/H3K9me3/HP1 skeleton, in early carcinogenesis, may decrease the activation of tumor suppressors such as p53 (24-26). To confirm this recruitment, we first examined the enrichment of H4K12ac in heterochromatin and euchromatin. We found that the levels of H4K12ac in non-tumor tissues did not vary across chromatin species (Figure 1). However, in tumors, particularly luminal tumors, there was a strong increase in H4K12ac levels, which were similar to those in healthy tissues of TNBC tumors (Figure 1). Knowing that H4K12 is a target for TIP60 (27), we investigated the co-localization of H4K12ac and TIP60 in the different chromatin species in breast tumors.

High co-localization of H4K12ac and TIP60 was observed in healthy tissues compared to tumors (Figure 2). However, a decrease in co-localization of H4K12ac and TIP60 was observed in Luminal A and TNBCs, whereas in intermediate Luminal B tumors there was an increase in this co-localization (Figure 2). These results could suggest that TIP60 was already present in the different chromatin species to deposit the H4K12ac mark because there was a higher co-localization of H4K12ac and TIP60 in the corresponding healthy tissues compared to the tumors. However, the increased levels of H4K12ac and the colocalization with TIP60 observed in Luminal B tumors compared to Luminal A and TNBCs could be the result of a concentration or recruitment of TIP60 to compensate for the progressive loss of the SUV39H1/H3K9me3/HP1 skeleton. This loss of $\mathrm{H} 4 \mathrm{~K} 12 \mathrm{ac}$ enrichment and colocalization with TIP60 in TNBCs is due to the progressive loss of TIP60 protein expression in these tumors observed by western blotting (Figure 4). The loss of TIP60 protein during breast cancer progression has been demonstrated (23). Under-expression of TIP60 and P400 mRNA was observed in Luminal tumors and over-expression in TNBCs (Figure 3). This could suggest that the increased expression of TIP60 RNA was disrupted at the translational level, resulting in reduced levels of the protein. This study showed that during carcinogenesis, TIP60 is highly solicited in heterochromatin but also in euchromatin probably to maintain chromatin stability and integrity. During the progression and depending on the aggressiveness of carcinogenesis, translational perturbation of TIP60 leads to its loss but also to the loss of the TIP60/P400/H4K12ac skeleton and chromatin instability.

To better understand this mechanism (Figure 5), TIP60 or $\mathrm{P} 400$ depletion could be induced in vitro in different breast cancer and non-cancer cell lines. This will allow us to examine how H4K12ac levels vary in the different species of chromatin after this depletion. The depletion of TIP60 or P400 will also allow us to investigate how TIP60 acts on P400 and vice versa. 


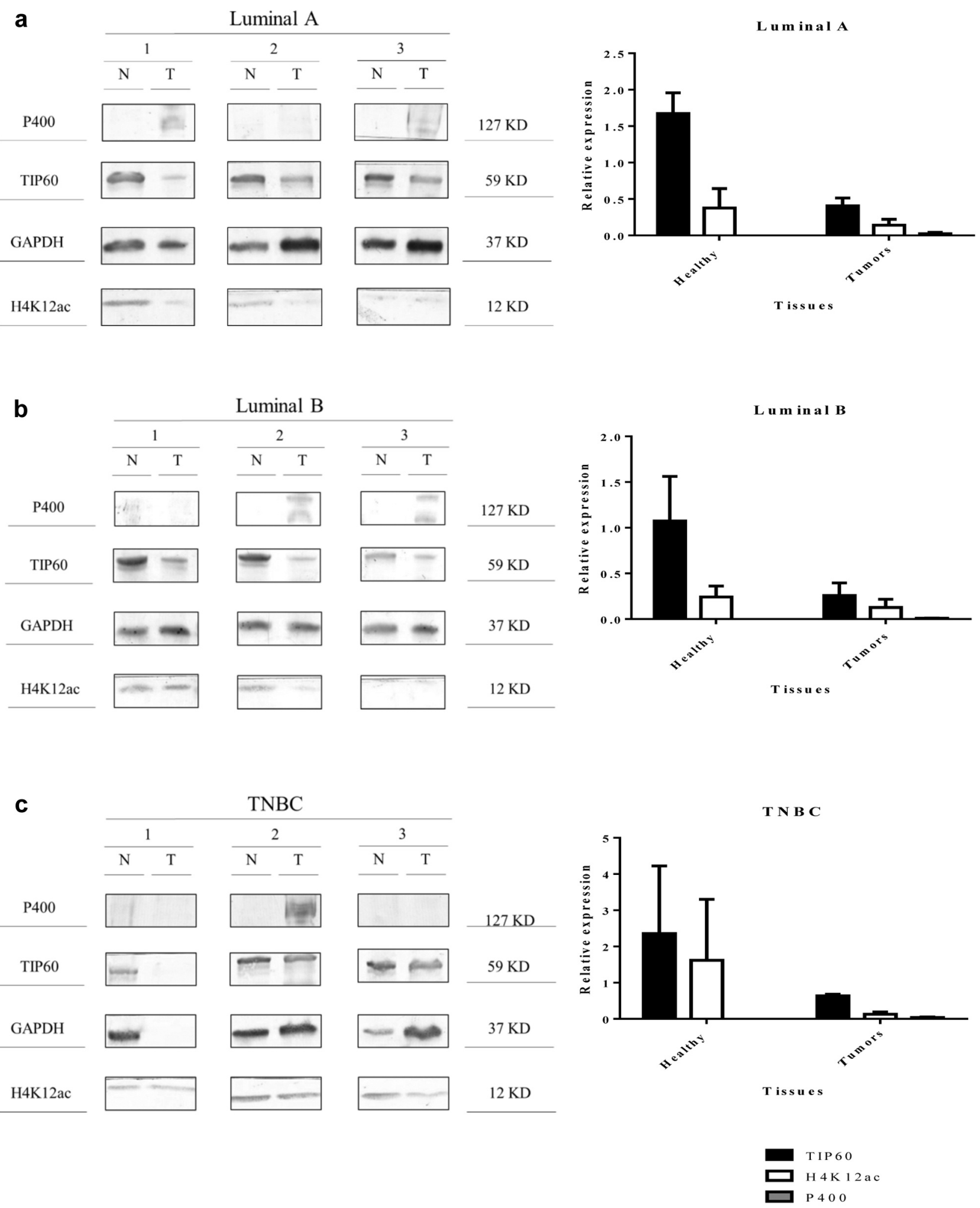

Figure 4. TIP60, P400, H4K12ac and GAPDH protein expression in the different breast tumors (Luminal A, Luminal B and TNBC) and their corresponding healthy adjacent tissues. (a) Shows the immunoblot of extracts from Luminal A breast tumors and their corresponding healthy tissues. (b) Shows the immunoblot of extracts from Luminal B breast tumors and their corresponding healthy tissues. (c) Shows the immunoblot of extracts from TNBC tumors and their corresponding healthy tissues. Quantification was performed by Image J. 


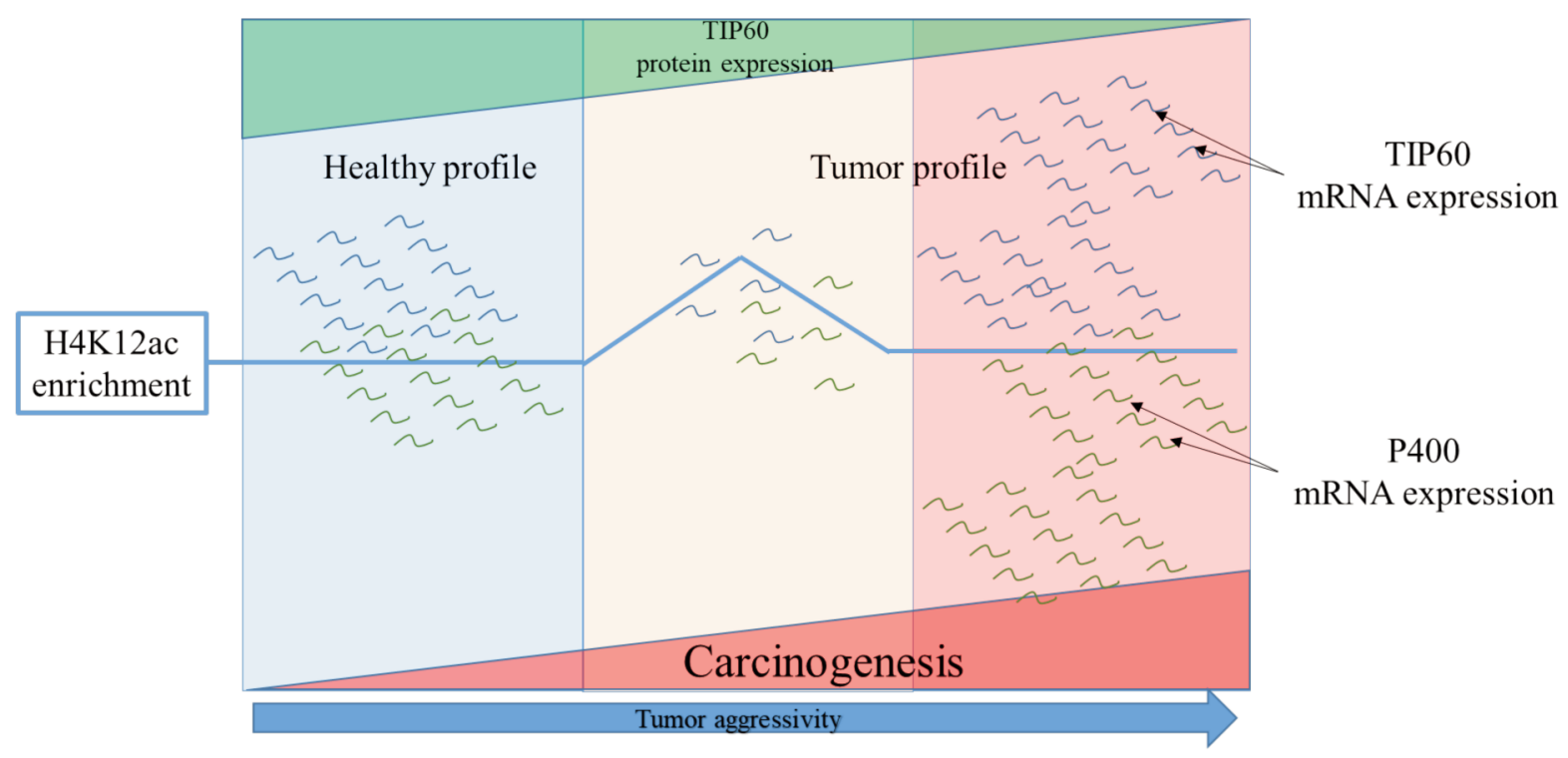

Figure 5. Illustration of the variation of H4K12ac enrichment as a function of tumor aggressiveness, protein expressions of TIP60 and P400 but also the expression of TIP60 and P400 RNA in heterochromatin and euchromatin.

\section{Conclusion}

In this study, the presence of TIP60 and H4K12ac modification was confirmed and a decrease of these two proteins was observed in breast tumors compared to healthy tissue. TIP60 and H4K12ac are present in euchromatin and heterochromatin. Finally, interaction between TIP60, P400 and H4K12ac was observed. The understanding of TIP60/P400/H4K12ac skeletal formation, the variations in its different components and the impact they have on each other will allow us to better understand carcinogenesis. This will undoubtedly allow us to use TIP60 or H4K12ac as a biomarker for the aggressiveness of the tumor, but also to use TIP60 as a therapeutic target against breast cancer.

\section{Conflicts of Interest}

There are no conflicts of interest to declare in relation to this study.

\section{Authors' Contributions}

Writing-review \& editing, Mouhamed Idrissou; Methodology, Tiphanie Boisnier, Anna Sanchez and Fatma Zohra Houfaf Khoufaf; Resources, Frédérique Penault-Llorca, Yves-Jean Bignon.; Supervision, project administration, Writing-review \& editing, Dominique Bernard-Gallon.

\section{Acknowledgements}

This work was supported by a grant from the French Ligue Régionale Contre Le Cancer - Comités du Puy-de-Dôme et de l'Allier.

\section{References}

1 Bray F, Ferlay J, Soerjomataram I, Siegel RL, Torre LA and Jemal A: Global cancer statistics 2018: Globocan estimates of incidence and mortality worldwide for 36 cancers in 185 countries. CA Cancer J Clin 68(6): 394-424, 2018. PMID: 30207593. DOI: $10.3322 /$ caac. 21492

2 Wooster R and Weber BL: Breast and ovarian cancer. N Engl J Med 348(23): 2339-2347, 2003. PMID: 12788999. DOI: 10.1056/ NEJMra012284

3 Lindstrom MS, Jurada D, Bursac S, Orsolic I, Bartek J and Volarevic S: Nucleolus as an emerging hub in maintenance of genome stability and cancer pathogenesis. Oncogene 37(18): 2351-2366, 2018. PMID: 29429989. DOI: 10.1038/s41388-0170121-z

4 Dawson MA and Kouzarides T: Cancer epigenetics: From mechanism to therapy. Cell 150(1): 12-27, 2012. PMID: 22770212. DOI: $10.1016 /$ j.cell.2012.06.013

5 Kanwal R, Gupta K and Gupta S: Cancer epigenetics: An introduction. Methods Mol Biol 1238: 3-25, 2015. PMID: 25421652. DOI: 10.1007/978-1-4939-1804-1_1

6 Nebbioso A, Tambaro FP, Dell'Aversana C and Altucci L: Cancer epigenetics: Moving forward. PLoS Genet 14(6): e1007362, 2018. PMID: 29879107. DOI: 10.1371/journal.pgen.1007362

7 Khanal P, Kim G, Lim SC, Yun HJ, Lee KY, Choi HK and Choi HS: Prolyl isomerase pin1 negatively regulates the stability of suv39h 1 to promote tumorigenesis in breast cancer. FASEB J 27(11): 4606-4618, 2013. PMID: 23934277. DOI: 10.1096/fj.13236851

8 Yokoyama Y, Hieda M, Nishioka Y, Matsumoto A, Higashi S, Kimura H, Yamamoto H, Mori M, Matsuura S and Matsuura N: Cancer-associated upregulation of histone h3 lysine 9 trimethylation promotes cell motility in vitro and drives tumor 
formation in vivo. Cancer Sci 104(7): 889-895, 2013. PMID: 23557258. DOI: $10.1111 /$ cas.12166

9 Allan RS, Zueva E, Cammas F, Schreiber HA, Masson V, Belz GT, Roche D, Maison C, Quivy JP, Almouzni G and Amigorena $\mathrm{S}$ : An epigenetic silencing pathway controlling t helper 2 cell lineage commitment. Nature 487(7406): 249-253, 2012. PMID: 22763435. DOI: $10.1038 /$ nature 11173

10 Kanwal R and Gupta S: Epigenetics and cancer. J Appl Physiol (1985) 109(2): 598-605, 2010. PMID: 20203073. DOI: 10.1152/japplphysiol.00066.2010

11 Ghobashi AH and Kamel MA: Tip60: Updates. J Appl Genet 59(2): 161-168, 2018. PMID: 29549519. DOI: 10.1007/s13353018-0432-y

12 Sun Y, Jiang X, Chen S, Fernandes N and Price BD: A role for the tip60 histone acetyltransferase in the acetylation and activation of atm. Proc Natl Acad Sci U S A 102(37): 1318213187, 2005. PMID: 16141325. DOI: 10.1073/pnas.0504211102

13 Wang P, Bao H, Zhang XP, Liu F and Wang W: Regulation of tip60-dependent p53 acetylation in cell fate decision. FEBS Lett 593(1): 13-22, 2019. PMID: 30414319. DOI: 10.1002/18733468.13287

14 Judes G, Rifai K, Ngollo M, Daures M, Bignon YJ, PenaultLlorca F and Bernard-Gallon D: A bivalent role of tip60 histone acetyl transferase in human cancer. Epigenomics 7(8): 13511363, 2015. PMID: 26638912. DOI: 10.2217/epi.15.76

15 Rajagopalan D, Tirado-Magallanes R, Bhatia SS, Teo WS, Sian S, Hora S, Lee KK, Zhang Y, Jadhav SP, Wu Y, Gan YH, Karnani N, Benoukraf $\mathrm{T}$ and Jha S: Tip60 represses activation of endogenous retroviral elements. Nucleic Acids Res 46(18): 9456-9470, 2018. PMID: 30053221. DOI: 10.1093/nar/gky659

16 Grezy A, Chevillard-Briet M, Trouche D and Escaffit F: Control of genetic stability by a new heterochromatin compaction pathway involving the tip60 histone acetyltransferase. Mol Biol Cell 27(4): 599-607, 2016. PMID: 26700317. DOI: 10.1091/mbc.E15-05-0316

17 Rangasamy D: Histone variant h2a.Z can serve as a new target for breast cancer therapy. Curr Med Chem 17(28): 3155-3161, 2010. PMID: 20666725. DOI: 10.2174/092986710792231941

18 Idrissou M, Rifai K, Daures M, Penault-Llorca F, Bignon YJ and Bernard-Gallon D: Exciting history of tip60 and its companions in carcinogenesis across the heterochromatin landscapes. OMICS 22(9): 626-628, 2018. PMID: 30106669. DOI: 10.1089/omi.2018.0122

19 Gorrini C, Squatrito M, Luise C, Syed N, Perna D, Wark L, Martinato F, Sardella D, Verrecchia A, Bennett S, Confalonieri S, Cesaroni M, Marchesi F, Gasco M, Scanziani E, Capra M, Mai S, Nuciforo P, Crook T, Lough J and Amati B: Tip60 is a haplo-insufficient tumour suppressor required for an oncogeneinduced DNA damage response. Nature 448(7157): 1063-1067, 2007. PMID: 17728759 . DOI: $10.1038 /$ nature06055
20 Rifai K, Judes G, Idrissou M, Daures M, Bignon YJ, PenaultLlorca $\mathrm{F}$ and Bernard-Gallon D: Sirt1-dependent epigenetic regulation of $\mathrm{h} 3$ and $\mathrm{h} 4$ histone acetylation in human breast cancer. Oncotarget 9(55): 30661-30678, 2018. PMID: 6078139. DOI: 10.18632 /oncotarget.25771

21 Judes G, Dubois L, Rifai K, Idrissou M, Mishellany F, Pajon A, Besse S, Daures M, Degoul F, Bignon YJ, Penault-Llorca F and Bernard-Gallon D: Tip60: An actor in acetylation of h3k4 and tumor development in breast cancer. Epigenomics 10(11): 14151430, 2018. PMID: 30324811. DOI: 10.2217/epi-2018-0004

22 Daures M, Idrissou M, Judes G, Rifai K, Penault-Llorca F, Bignon YJ, Guy L and Bernard-Gallon D: A new metabolic gene signature in prostate cancer regulated by jmjd3 and ezh2. Oncotarget 9(34): 23413-23425, 2018. PMID: 5955128. DOI: 10.18632/oncotarget.25182

23 Mattera L, Escaffit F, Pillaire MJ, Selves J, Tyteca S, Hoffmann JS, Gourraud PA, Chevillard-Briet M, Cazaux C and Trouche D: The p400/tip60 ratio is critical for colorectal cancer cell proliferation through DNA damage response pathways. Oncogene 28(12): 1506-1517, 2009. PMID: 19169279. DOI: 10.1038/onc. 2008.499

24 Tang Y, Luo J, Zhang W and Gu W: Tip60-dependent acetylation of p53 modulates the decision between cell-cycle arrest and apoptosis. Mol Cell 24(6): 827-839, 2006. PMID: 17189186. DOI: 10.1016/j.molcel.2006.11.021

25 Bassi C, Li YT, Khu K, Mateo F, Baniasadi PS, Elia A, Mason J, Stambolic V, Pujana MA, Mak TW and Gorrini C: The acetyltransferase tip60 contributes to mammary tumorigenesis by modulating DNA repair. Cell Death Differ 23(7): 1198-1208, 2016. PMID: 4946888. DOI: $10.1038 /$ cdd.2015.173

26 Dar A, Shibata E and Dutta A: Deubiquitination of tip60 by usp7 determines the activity of the p53-dependent apoptotic pathway. Mol Cell Biol 33(16): 3309-3320, 2013. PMID: 3753911. DOI: 10.1128/MCB.00358-13

27 Kimura A and Horikoshi M: Tip60 acetylates six lysines of a specific class in core histones in vitro. Genes Cells 3(12): 789-800, 1998. PMID: 10096020. DOI: 10.1046/j.1365-2443.1998.00229.x

Received August 26, 2020

Revised September 8, 2020 Accepted September 10, 2020 